\title{
Recent advances in FT-IR (Fourier transform infrared spectroscopy) of polymers
}

\author{
Jack L. Koenig \\ Department of Macromolecular Science,Case Western Reserve University, \\ University Circle, Cleveland, Ohio 44106

\begin{abstract}
Recent developments in FT-IR sampling techniques such as diffuse reflectance and photoacoustic spectroscopy are known to obtain new and novel information on solid polymers. In particular, depth profiling can be obtained from the surface using a potassium bromide overlayer. Photoacoustic spectroscopy using coupling gases with different polarizability allows one to determine the nature of absorbed gases on polymer surfaces.
\end{abstract}

\section{INTRODUCTION}

Fourier Transform Infrared spectroscopy(FT-IR) is developing as a ubquitous tool for use in the characterization of polymers(1). This paper will review some of the recent advances. Historically, one can observe three stages in the development of FT-IR.

First, there was the stage of instrument development where interferometers were being designed for the infrared region and minicomputers were being utilized for the Fourier computations. This stage produced commercial instruments capable of obtaining spectra of polymers with higher signal to noise ratios than were possible with the dispersion infrared equipment(2). However, the utility of the higher signal to noise ratio was not initially particularly useful for polymeric spectra.

The second stage of development of FT-IR could be termed the data processing stage (3). In this stage, the dedicated minicomputer on the FT-IR instrumentation allowed one to manipulate the spectra data and particularly important was absorbance subtraction(4) for comparing two spectra. Difference spectroscopy was especially valuable for polymers since the subtraction procedure eliminated the interfereing absorbance of the polymer itself. With the elimination of this interference, the subtle changes induced by chemical reactions, environmental effects and stress could be observed. However, at this stage in the development of FT-IR, the primary restriction in utility of FT-IR for polymers resided in the sampling techniques.

The third stage of development of FT-IR involved the evolution of sampling techniques which not only simplified the experiment but allowed one to obtain new information (5). With the high energy througput, it has been possible to utilize a number of sampling techniques that were unavailable in the infrared region prior to FT-IR. Diffuse reflectance spectrscopy has had a long history in the ultraviolet and visible region but has only recently been extended to the infrared(6) and has been termed DRIFT. Simililarly, photoacoustic spectroscopy (PAS) which was orignated by Graham Bell, has only recently been used in the FT-IR(7). This paper will outline recent advances in using these two techniques for the study of polymeric materials.

\section{DEVELOPMENT OF A DEPTH PROFILING TECHNIQUE USING DIFFUSE REFLECTANCE} TECHNIQUES

A recent development in the use of DRIFT is the discovery of a simple spectroscopic method of depth profiling the surfaces and interfaces of organic/inorganic materials $(8,9,10)$. The method is based on applying diffuse reflectance spectroscopic techniques using Fourier transform infrared instrumentation to bulk materials rather than to powdered materials for which the DRIFT technique is specifically designed.

The new observation involves the use of a powdered potassium bromide(KBr) overlayer on top of the bulk sample to be examined. As the overlayer thickness is increased, the surface/bulk contribution to the recorded spectrum increases. The reflected beam which is observed at the detector contains contributions from the various regions and using suitable 
data prucessing techniques it is possible to determine the relative contributions of the surface and bulk spectra to the recorded spectra. This makes possible quantitative determinations of differences in structure as a function of depth from the reflecting surface.

The applicability of the $\mathrm{KBr}$ overlayer technique to bulk organic materials was first demonstrated(8). We have made some measurements using this technique involving a poly (vinylidene flouride) ( $\mathrm{PVF}_{2}$ ) overlayer on a polyethylene terephthalate(PET) substrate (Figure 1). We were able to quantitate the relative contributions of $\mathrm{PVF}_{2} / \mathrm{PET}$ and effectively separate the spectrum of the thin $(200 \mathrm{~A})$ upperlayer from the substrate (Figure 2$)$. The type of results obtained using this type of approach for a coupling agent deposited on glass mats is shown in Figure $3(10)$. A small circular mat of sample was covered with a uniform layer of $\mathrm{KBr}$. At the bottom of Figure 3, we show the spectrum of the heat cleaned E-glass fiber. Spectrum (B) is the DRIFT spectrum of E-glass fiber treated with a 38 by weight solution of $\gamma$-aminopropyltriethoxysilane $(\gamma-A P S)$. Note that relative to the spectrum of the uncovered E-glass fiber/coupling agent sample, the intensity of the E-glass bands are reduced by a factor of 10 . The difference spectrum(C) in Figure 3 is the result of subtracting spectrum A from spectrum B and is representative of the spectrum of $\gamma$-APS. One observes that the difference spectrum is precisely that of $\gamma$-APS. Although it is not shown here the spectrum with the $\mathrm{KBr}$ overlayer yields a spectrum which is virtually identical to the coupling agent. Spectra from the thinner layers of the $\mathrm{KBr}$ overlayer exhibit contributions from the glass substrate. Using suitable data processing techniques it is possible to determine the relative contributions of the surface and bulk spectra to the recorded spectra.

The technique yields useful results in depth profiling of surfaces and interfaces, but, to date, we do not have a clear understanding of the optical physics involved. Apparently, the additional scattering induced by the $\mathrm{KBr}$ overlayer restricts the penetration of the infrared beam into the bulk material. Since the diffuse reflectance attachments in the infrared are not optically perfect, that is, the incoming light is not completely diffuse, it is possible that the $\mathrm{KBr}$ overlayer is changing the average angle of reflection which could result in lesser penetration. This is the basis for depth profiling using ATR and external specular reflection. We are still investigating this aspect of the problem and would like a better understanding of the optics involved as it would possibly help in optimizing the use of the $\mathrm{KBr}$ overlayer technique in diffuse reflectance spectroscopy as a depth profiling method.

\section{DEVELOPMENT OF A DEPTH PROFILING TECHNIQUE USING PHOTOACOUSTIC SPECTRSCOPY}

Photoacoustic spectroscopy(PAS) in the infrared region has potential for depth profiling of organic materials (11). The basis of photoacoustic spectroscopy is illustrated in Figure 4. In figure 4, a PAS sample cell is depicted which is illuminated by a beam of light chopped at an audiofrequency. The light beam passes through a transparent gas behind the window and is incident upon the solid sample. Energy absorbed by the sample surface from the incident light may be converted by radiationless transitions to a thermal wave that returns (by thermal diffusion) and warms the thin layer of gas in contact with the surface. This layer of periodically heated gas acts as a "thermal piston" on the rest of the gas in the cell and causes a sound wave of the same frequency as that at which the light beam was chopped but of delayed phase. These acoustic waves are detected by the microphone and transformed into the photoacoustic spectra. The photoacoustic signal intensity $Q$ is given by the proportionality

$$
Q=a \text { u } f^{-3 / 2} \text {, }
$$

where $a$ is the optical absorptivity $\left(\mathrm{cm}^{-1}\right), u$ is the thermal diffusion length $(\mathrm{cm})$ and $f$ is the beam chopping frequency $\left(\mathrm{s}^{-1}\right)$. For Fourier transform spectroscopy the beam chopping is determined by the motion of the moving mirror and the frequency is given by

$$
\mathbf{f}=2 \mathrm{v} \mathbf{w}
$$

where $\mathrm{v}$ is the mirror speed $\left(\mathrm{cm} \mathrm{s}^{-1}\right)$ and $\mathrm{w}$ is the infrared frequency $\left(\mathrm{cm}^{-1}\right)$.

A second consideration favoring PAS is that the specular scattered light from the sample is not detected as in reflection techniques. The scattered light reduces the dynamic range of the spectroscopic response and 


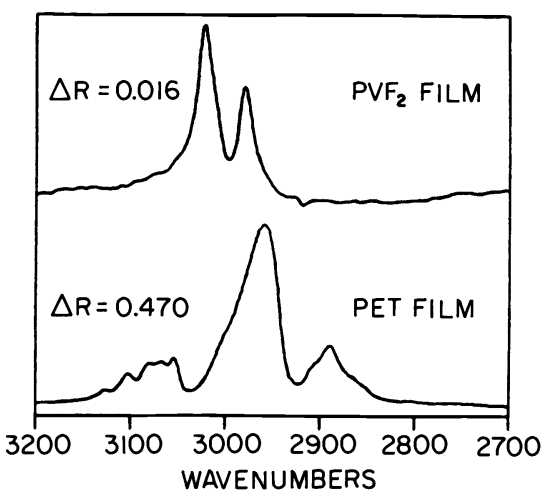

Fig. 1. Diffuse reflectance spectra of PVF and PET films in the 3200 $2700 \mathrm{~cm}^{-1}$ region.

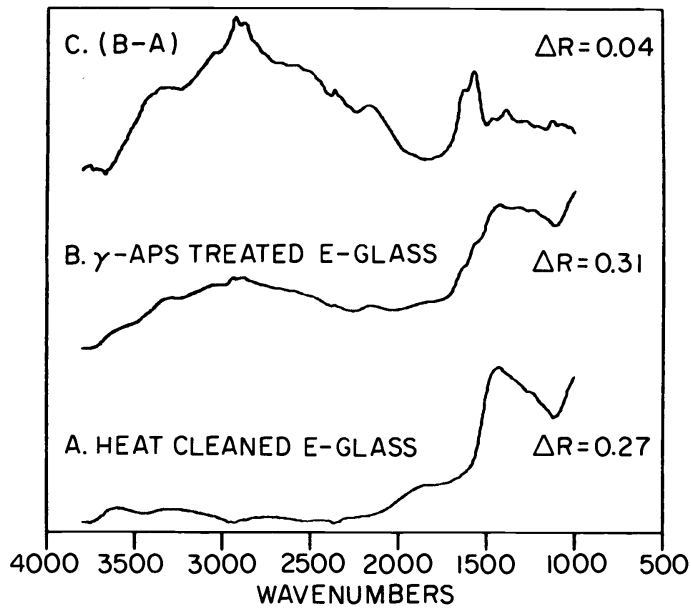

Fig. 3. DRIFT spectra of (A) heat cleaned glass, (B) $\gamma$-treated E-glass, (C) Diffuse spectrum.

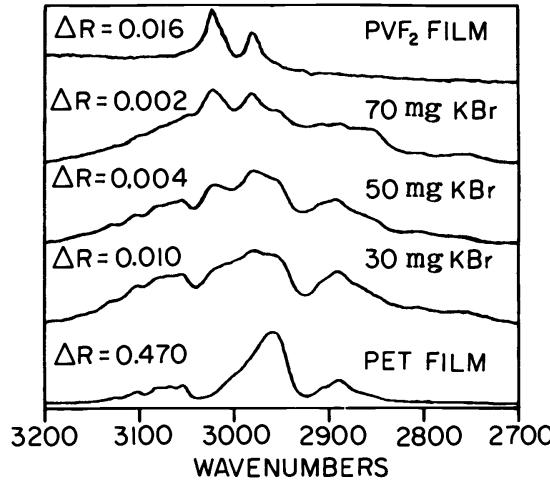

Fig. 2. DRIFT spectra of $\mathrm{PVF}_{2} / \mathrm{PET}$ sandwich with increasing amounts of $\mathrm{KBr}$.

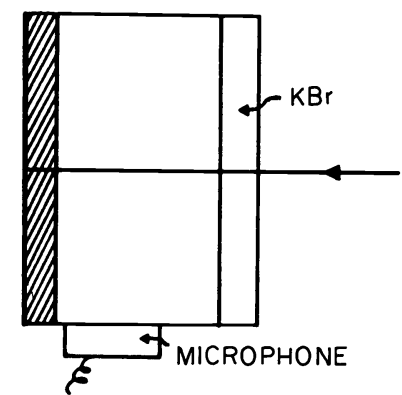

Fig. 4. Diagram of Photoacoustic cell.

lowers the contrast and intensity of the resultant infrared spectrum. A final consideration is the use of PAS to study opaque samples. Obviously, no requirement of light transmission is imposed on the sample for measurement by PAS so highly absorbing samples can be utilized (12).

However, PAS has some serious limitations when quantitative measurments are being made. Two factors are important, saturation and scattering. Saturation occurs when the optical absorbance length approaches the thermal diffusion length. However, if proper care is taken saturation can be minimized. Scattering, on the other hand, limits the signal intensity and with high scattering, additional scans must be taken to overcome the loss of signal. Since PAS has a low initial signal-to-noise ratio, this additional scanning time may be prohibitive. However, PAS may be the only suitable sampling technique for samples where it is necessary to preserve the thermal, processing or mechanical history. Under these circumstances, PAS should be tested.

As a surface probe PAS has a special advantage over other techniques, that is, since it is an enclosed system it is possible to simultaneously determine the amount of gas phase species and adsorbed species. In this way, one can determine the amount of absorption on a surface. From such a measurement it may be possible to calculate the fraction of active sites. Measurements of this kind have been made using PAs to study the absorption of $\mathrm{CO}$ on an opaque $\mathrm{Ni} / \mathrm{SiO}_{2}$ heterogeneous catalyst (13). For such measurments it is necessary to construct a calibration curve of PAS response to the gas in the absence of the substrate. Then injecting a known amount of gas in the presence of the substrate allows one to measure the amount of gas absorbed on the surface. Since the spectrum of the adsorbed species is recorded, it is possible to detect the sorbed species 
simultaneously. Comparison of the spectra of the substrate before and after adsorption of the gas allows the determination of the nature of the "active" sites.

We have utilized a novel approach to PAS based on the theory of Dignam, Rao and Roth (14). This theory was not proposed for PAS, because at the time the theory was generated, it was not possible to do PAS in the infrared region. However, the extension of the theory to PAS is obvious. The theory suggests that if a highly polarizable gas is adsorbed on a surface, those surface species with a transition moment parallel to the surface will exhibit enhanced absorption while those with a transition moment perpendicular to the surface will be suppressed. This theory was used to interpret early infared data for hydroxyl groups on silica (15).

In our laboratory we have pursued this approach using PAS where we have exchanged the helium coupling gas with xenon. Figure 5 shows the FT-IR PAS spectra of silica in the 4000-500 $\mathrm{cm}^{-1}$ region. Trace $A$ is obtained on the silica sample with helium as the coupling gas and trace $B$ is obtained after the helium has been purged and replaced with xenon. Trace $C$ was obtained by purging the zenon and replacing with helium. The latter trace was obtained to determine that no irreversible effects had occured and that Trace $A$ and Trace $C$ are virtually identical. Comparison of the spectra with the He and Xe coupling gases shows some striking differences. The first observation is the large modification of the relative intensities in different regions of the spectra. The $O H$ stretching region showing the free $\mathrm{OH}$ at $3745 \mathrm{~cm}^{-1}$ and the broad hydrogen bonded water band 3670 to 3440 $\mathrm{cm}^{-1}$ are highly suppressed with the xenon as the coupling gas. The Dignam theory would suggest this occurs because the $O H$ groups on the silica surface and the adsorbed water are predominately normal to the surface. On the other hand, the region involving the bands at 2200,1991 and $1838 \mathrm{~cm}^{-1}$ are much enhanced with the xenon coupling gas. This region of the spectrum is usually associated with carbon monoxide adsorption. The very high enhancement suggests that the carbon monoxide is aligned parallel with the surface so the highly polarizable xenon causes a change in the polarization of the molecule on the surface.

\section{SPECTROSCOPIC CHARACTERIZATION OF THE INTERFACE IN ORGANIC FIBER COMPOSITES}

We have applied the above techniques to a study of the surfaces of Kevlar fibers. To obtain the optimum strength properties of an organic fiber composite, it is necessary to have good adhesion between the fiber and the matrix. For fibers of poly (p-phenylene terephthalamide)(PPT) which are commercially produced as Kevlar $R$ by Dupont and known commonly as polyaramid fibers, this adhesion is highly limited (16)(17)(18). The chemical inertness which leads to the excellent chemical stability does not allow interfacial reactions with the polymeric matrix. Consequently, it may be necessary to prepare the fiber surface by chemical reactions or to make use of adhesion promoters such as coupling agents. In addition, PPT may reversibly absorb as much as 2 to 38 moisture when exposed to high humidity at ambient temperatures(19). This water sorption could act to weaken interfacial adhesion to polymeric matrices.

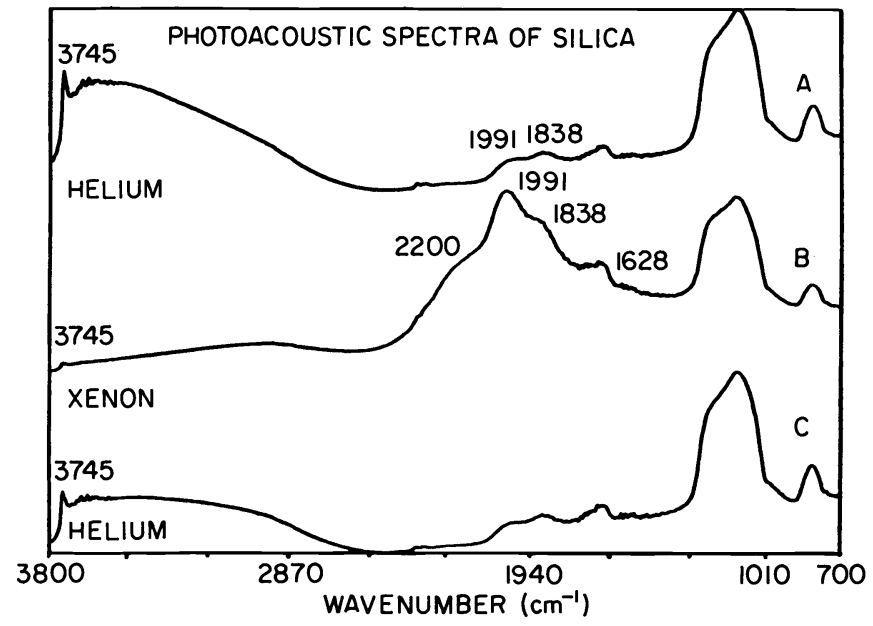

Fig. 5. FT-IR PA spectra of silica in the $4000-500 \mathrm{~cm}^{-1}$ region. Trace A-coupling gas: helium; trace B-coupling gas: xenon; trace C-coupling gas: helium. 
The first step in adhesion improvement is a knowledge of the surface functionality of the fiber. The polyamide fiber is prepared by polymerizing the acid chloride of terephthalic acid with p-phenylenediamine in a suitable solvent (20)(21)(22)(23). The polymer is dissoved in sulfuric acid and, from an oriented solution, is formed into fiber filaments with a dry-jet wet spinning process. Under these circumstances, few endgroups are expected and the aromatic structure responsible for the chain rigidity sterically hinders the amide groups from interfacial reactions. However, due to the complexity of the synthesis and spinning it is necessary to determine precisely the surface functionality of the fiber.

Prior to FT-IR determinations, one must remove any fiber spinning aids, additives, greases, or other surface impurities present as a result of fiber formation, spinning, weaving and general handling. These impurities can be removed by solvent extraction of the Kevlar fibers. A procedure for cleaning of the fibers has been described (24), involving a soxhlet refluxing of the fibers in 1,2-dichloroethane for 24 hours followed by a reflux in methanol and water for a similar period of time.

PAS FT-IR spectra of as-received fibers of Kevlar are shown in figure 6. Spectrum $A$ was obtained using xenon and spectrum $B$ using helium as a coupling gas in the photoacoustic cell. The difference spectrum (A-B) obtained by using a scaling coefficient calculated by least-squares fitting of $A$ and $B$ in the region between 2000 and $700 \mathrm{~cm}^{-1}$, is shown in trace $C$. The spectrum with the highly polar intert gas zenon shows enhanced absorbance of the aromatic $\mathrm{C}-\mathrm{H}$ stretching band at $3043 \mathrm{~cm}^{-1}$, as well as some enhancement of the amide $\mathrm{N}-\mathrm{H}$ stretching bands at 3327 and $3145 \mathrm{~cm}^{-1}$. This result indicates that both the aromatic rings and the amide groups are essentially oriented parallel to the surface of the fibers. The broad water band at about $3600 \mathrm{~cm}^{-1}$ is not enhanced by the use of xenon as a coupling gas, meaning that the chemisorbed water is either randomly oriented on the surface of the fibers or not present on the fibers, that is, it has diffused into the skin of the fiber.

Similiar spectra of dried fibers show that the adsorption of moisture by the fibers does not affect the orientatioin of the aromatic rings. The amide $\mathrm{N}-\mathrm{H}$ stretching bands are more enhanced in xenon than the aromatic $\mathrm{C}-\mathrm{H}$ stretching peak.

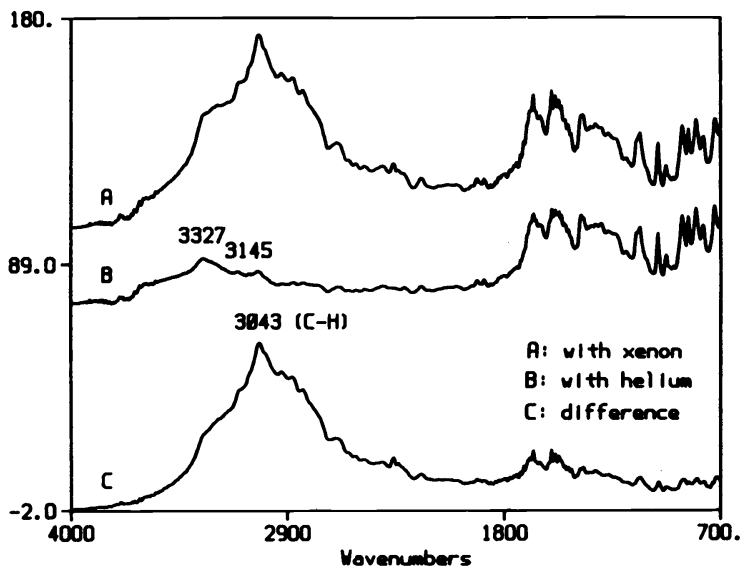

Fig. 6. Photoacoustic spectra of as-received Kevlar $-49^{\mathrm{R}}$ with: (A) coupling gas xenon, (B) coupling gas helium, (C) difference (A-B).

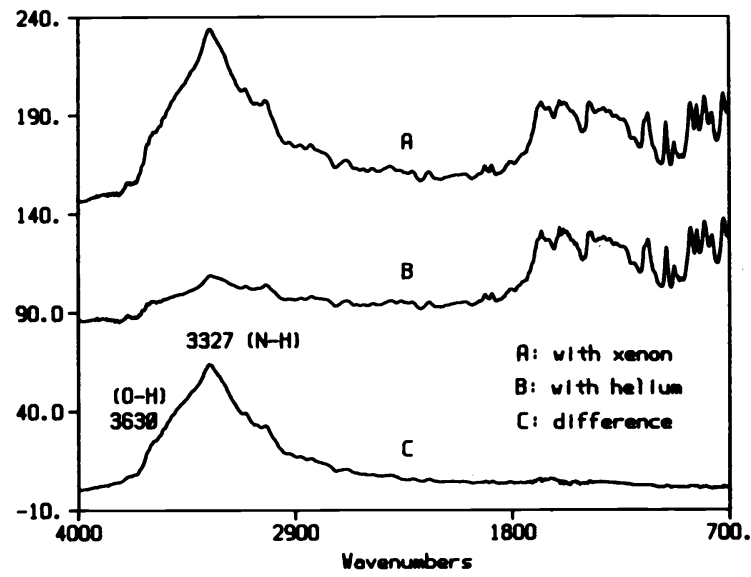

Fig. 7. Photoacoustic spectra of Kevlar $-49^{\mathrm{R}}$ fibers hydrolyzed for $3 \mathrm{hrs}$. in boiling $\mathrm{H}_{2} \mathrm{SO}_{4}$ solution (N 10) with (A) coupling gas xenon, (B) coupling gas helium, (C) difference (A-B).

These data can be interpreted if we visualize the dried fibers as having the amide groups lying completely parallel to the surface and the benzene rings having internal rotational alngles of possibly 300 measured from the amide plane. This implies that the major component of the $\mathrm{C}-\mathrm{H}$ dipole moment is parallel to the fiber surface. When the fibers adsorb moisture, the water molecules form hydrogen bonds with the surface $\mathrm{N}-\mathrm{H}$ groups, possibly substituting some of the intermolecular hydrogen-bonds between adjacent amides. Thus the orientation of the $\mathrm{N}-\mathrm{H}$ bonds changes from completely parallel to tilted with respect to the surface of the fibers.

The PAS spectra of hydrolyzed Kevlar-49 fibers in xenon and helium are shown in traces $A$ and $B$, respectively, in Figure 7. Spectrum $B$ was subtracted from A using the scaling coefficient obtained by a least-squares 
fitting program applied for the region $2000-700 \mathrm{~cm}^{-1}$. The difference, trace $C$ of Figure 7, shows that the polar xenon enhances the amide $\mathrm{N}-\mathrm{H}$ stretching band at $3327 \mathrm{~cm}^{-1}$, thus indicating that there is a preferred orientation of the $\mathrm{N}-\mathrm{H}$ groups, parallel to the surface. In addition, a broad and intense $0-H$ stretching band at about $3600 \mathrm{~cm}^{-1}$ is enhanced indicating the presence of chemisorbed water on the surface. The hydrolysis procedure removes the skin leaving the core exposed. So these orientation differences are attributed to the differences in the structure of the skin and the core of the fibers. The aromatic rings in the skin are oriented almost parallel to the surface of the fibers whereas the $\mathrm{N}-\mathrm{H}$ bonds of the amide groups of the core are parallel to the fiber surfaces. These observations suggest the structure of the core has extensive intermolecular hydrogen-bonds between the amide groups, with the amide N-H's radially oriented. The plane of the aromatic rings of the chains is also in the radial direction, forming $300^{\circ}$ rotational angles relative to the amide plane.

\section{SUMMARY AND CONCLUSIONS}

Two conclusions can be drawn from these PAS experiments. First, the change of from a nonpolar to polar coupling gas produces a large enhancement of the adsorption of some of the surface species. This conclusion leads to improved sensitivity of PAs for surface analysis. Secondly, the relative orientation of the surface species with respect to the surface can be obtained by measuring either the enhancement or suppression of the absorbance relative to the same sample using helium as a coupling gas. This latter measurement of the orientation of the surface species is an illusive factor by another method. The PAS approach is simple and apparently quite powerful in this regard.

It is apparent from the above results that the utility of FT-IR relative to polymer characterization is rapidly being developed in terms of simplicity of sampling and type of information which is obtained..

\section{REFERENCES}

(1) J.L. Koenig, "Fourier Transform Spectroscopy of Polymers" in Advances in Polymer Science, 54, 87, Springer-Verlag Publ. 1983.

(2) P. R. Griffiths Chemical Infrared Transform Spectroscopy, Wiley, New York, 1975

(3) J.L. Koenig, Pure and Appl. Chem. 54(2), 439-446, 1982,

(4) J. L. Koenig, D. L. Tabb, M. M. Coleman, J. Polym. Sci., Polym. Phys. Ed. 13,1145 (1975)

(5) S.R. Culler, H. Ishida, and J.L. Koenig, Ann. Rev. Mater.Sci., 13, 363-386 (1983).

(6) M. P. Fuller and P. R. Griffiths, Anal. Chem. 50, 1906 (1978)

(7) M. G. Rockley Chem. Phys. Lett., 68, 455 (1979)

(8) S.R. Culler, M.T. McKenzie, L.J. Fina, H. Ishida, and J.L. Koenig, Appl. Spectrosc 38791 (1984)

(9) M.T. McKenzie and J.L. Koenig, "Further Developments in the Methodology of Surface Analysis by FT-IR: Quantitative Aspects of Diffuse Reflectance Methods" Appl. Spectrosc. (in press).

(10) M. T. McKenzie, S.R. Culler, and J.L. Koenig, Appl. Spectrosc. 38 786 (1984)

(11) K. Krishnan, Appl. Spectrosc. 35. 549 (1981)

(12) A. Rosencwaig, Photoacoustics and Photoacoustic spectroscopy, (John Wiley and Sons, New York, 1980)

(13) J. A. Gardella Jr., Da-Zhen Jiang and E. M. Eyring, Appl. Spectrosc. 37, 131 (1983)

(14) M. J. Dignam, B. Rao and J. R. Roth, J. Chem. Soc Faraday Trans II, 69,80 (1973)

(15) R. S. McDonald, J. Amer. Chem. Soc., 79850 (1957)

(16) W. B. Black and J. Preston, "High Modulus Wholly Aromatic Fibers, Marcel Dekker, New York, 1973

(17) L. Konopasek and J. W. S. Hearle, J. Appl. Sci., 212791 (1977)

(18) 0. B Eagles, B. F. Blumentritt and S. L. Cooper J. Appl. Polymer Sci., 20, 435 (1976)

(19) L. Penn and F. Larsen, J. Appl. Polymer Sci., 23, 59 (1979)

(20) S. L. Kwolek, P. W. Morgan and W. R. Sorensen, U. S. Pat. 3,063,966 (November 13, 1962)

(21) S. L. Kwolek, U. S. Pat. 3,600,350 (August 17, 1971)

(22) S. L. Kwolek, U. S. Pat. 3,671,542 (June 20, 1972)

(23) H. Blades, U.S. Pat. 3,767,756 (Octomber 23, 1973)

(24) D. J. Vaughan in Proc. llth Natl. SAMPE Conf. Held Nov. 13-15, 1979; pp. 593 